\title{
Total energy consumption analysis in wireless mobile ad hoc network with varying mobile nodes
}

\author{
Mohammed A. Saad ${ }^{1}$, Sameer A. S. Lafta ${ }^{2}$, Raed Khalid Al-Azzawi ${ }^{3}$, Adnan H. Ali ${ }^{4}$, Sameer Alani ${ }^{5}$, \\ Mohammed M. Hashim ${ }^{6}$, Bassam Hasan ${ }^{7}$ \\ ${ }^{1}$ Department of Medical Instrumentations Technique Engineering, Alkitab University, Kirkuk, Iraq \\ ${ }^{1}$ Faculty of Engineering \& Built Environment, University Kebangsaan Malaysia (UKM), Malaysia \\ ${ }^{2}$ Middle Technical University, Technical Instructors Training Institute, Baghdad, Iraq \\ ${ }^{3}$ Al-Farahidi University, Baghdad, Iraq \\ ${ }^{4}$ Middle Technical University, Institute of Technology-Baghdad, Iraq \\ ${ }^{5}$ Department of Computer Technical Engineering, Al-Kitab University College, Kirkuk, Alton Kupri, Iraq \\ ${ }^{6}$ Faculty of Engineering, Uruk University, Baghdad, Iraq \\ ${ }^{6}$ Technical Engineering College, Middle Technical University, Baghdad, Iraq \\ ${ }^{7}$ Department of Electronics and Communication Engineering, College of Engineering, Universiti Tenaga Nasional, \\ Malaysia
}

\begin{tabular}{l}
\hline Article Info \\
\hline Article history: \\
Received Aug 26, 2019 \\
Revised Jan 28, 2020 \\
Accepted May 6, 2020
\end{tabular}

\section{Keywords:}

Energy consumption

MANET

Mobile ad-hoc networks

NS-3

OLSR, AODV, DSR

Wireless network

\begin{abstract}
The energy protocols that have a mechanisms of shortest path routing considered predominant in the networking scenarios. The interesting matter in the routing protocols designing deal with mobile ad hoc network (MANET) must have an energy efficient network for better network performances. The Performances of such routing protocols that can be assessed will be focused on many metrics like delay, throughput, and packet delivery. MANET is a distribution network, having no infrastructure and network decentralization. There routing protocols are utilized for detecting paths among mobile nodes to simplify network communication. The performance comparison of three protocols are optimized link state routing (OLSR), the second is ad hoc on-demand distance vector (AODV), while the third is dynamic source routing (DSR) routing protocols concerning to average energy consumption and mobile node numbers are described thoroughly by NS-3 simulator. The nodes number is changing between 10 and 25 nodes, with various mobility models. The performance analysis shows that the suggested protocols are superior in relations to the energy consumption for networking data transmission and the performance of the wireless network can be improved greatly.
\end{abstract}

Copyright $\left({ }^{\circ} 2020\right.$ Institute of Advanced Engineering and Science. All rights reserved.

\section{Corresponding Author:}

Mohammed Ayad Saad

Faculty of Engineering \& Built Environment, University Kebangsaan Malaysia (UKM)

43000 Kajang Malaysia

Email: mohmmad9alani@gmail.com

\section{INTRODUCTION}

Wireless Local Area Network (WLAN) can be considred as a network which permits for interconnecting many devices with a distributed wireless techniques at a fixed area. This connection gives the clients more capability for moving around the coverage area so that to preserve a network connection. Modern WLANs use IEEE 802.11 standards as a basis, and referred commonly as Wi-Fi brand name [1,2]. The major benefit that performs being ability for Internet network connection, generally with external connection, besides an absence of wire limitation, gives it the most common using everywhere [3, 4]. MANET is a nodes collection which close together for dynamically network creating, with no fixed 
infrastructure or localized management [5], for a source that want data packets sending to a destination that not directly related to the transmitted range, thus, packets must be transmitted by one or more midway nodes.

Mobile networking considers a most developing universal computing technologies and has classically two groups of wireless networks concerning the infrastructure that are based and infrastructure less. Based infrastructure involves access points (base stations) for giving assistance to communicate, whereas no infrastructure needs no such devices or technologies and referred to ad hoc network. The wireless networking with multi hop at any forms are beyond to this type like mobile ad hoc network (MANET) and vehicular ad hoc network (VANET), wireless mesh network (WMN), and specially wireless sensor network (WSN) [6]. The greatest essential problem deals with ad hoc network focusing on professionally sending data packets to the mobile nodes. Subsequently node's topology modifies considerably which gives routing very challenging. Correspondingly, low bandwidth with a restricted battery ability and error expanded medium enhances more complexity in the well-organized routing protocols designing $[7,8]$.

The appearance of wireless mobile ad-hoc network (MANET) gives an efficient technique with more significantly cost-effective to create mobile hosts availability if infrastructure-less is providing. With MANET, a technique of easily communicating of mobile nodes with each other even though freely moving around at various directions [9]. The forward data information from the sources to proposed destination nodes in MANET relies totally on nodes cooperation [10]. Researchers focus their interesting on securing MANETs. Many methods were suggested for reducing or preventing the attack risks on the mobile ad-hoc networks with utilizing management of access control keys and mechanisms of trust models [11, 12]. The consumed energy is an important matter in MANET since the power of mobile nodes are with limited battery resources. Accordingly, network lifetime depends seriously on the consumed nodes energy. The energy saving is very critical for lifetime prolonging at any network. The MANET architecture can be shown in Figure 1.

The connection devices to a WLAN network are referred by stations or STAs, with a two types classifying: Access Points (APs), as well as Basic Stations (BSs) [13]. The presentation of APs are always didn't in all WLANs while they are typically wireless routers that can be forward packets to BSs, and make interconnection to another network. BSs are generally like mobile phones or may be laptops that can sending and receiving traffic data with further BSs or adjoining networks [14]. A set of stations that can communicating jointly can be termed Basic Service Set (BSS), with two types as in Figure 2: infrastructure BSS, and the other is independent BSS like ad hoc network, that comprises of no APs and has routing functionalities in BSs [15].

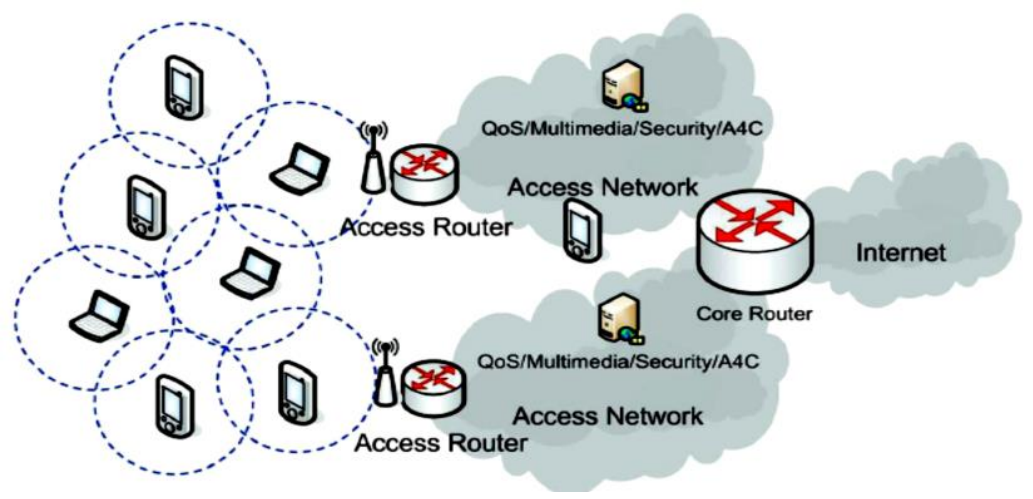

Figure 1. The MANET architecture

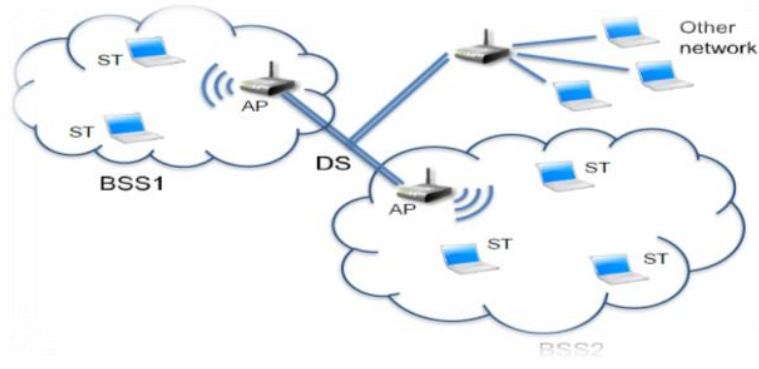

Figure2. BSS of Infrastructure WLANs 
The proposed paper focuses on evaluating and analyzing the consumed energy deals with three distinguished routing protocols in MANET under fixed bit rate traffic with respect to different mobile nodes number. The total energy consumption by each node throughout the action of transmitted and receipted traffic data have been assessed with a function of nodes number in the network.

\section{ROUTING PROTOCOLS IN AD-HOC}

Several routing protocols designed to ad-hoc networks will be suggested here, these protocols are running at the network as well as MAC layer. There are two major fundamentals of routing protocols in MANET, the route selection and distributed data packets precisely for the exact node. The routing protocols describe them by how discovering the packet routes and how sending these packets in the network. The most three routing protocols that can be chosen to compare are: reactive protocol or (on-demand) represented by AODV (ad hoc on-demand distance vector), proactive protocol or (table-driven) like OLSR (optimized link state routing protocol) and DSR (dynamic source routing), and the hybrid protocol characteristic with HWMP (hybrid wireless mesh protocol) [16, 17]. A selection had been done from long list of routing protocols that have acceptance and complete implementation in simulator [18].

\subsection{Table-driven routing protocols (proactive)}

Table-based protocols permanently attempt to maintain the relevance and consistency of routing data for each network node. These protocols try to preserve exact routing information for the entire network at any time. Each network's node supports routing information with supporting many routing tables. Information activate about the target nodes usually done with the nodes attempting by routing tables updating. The adaption of routing protocol for sudden changings in the structure, and transmits network updates every time changes occur. Proactive routing protocols will not be waiting for the request before looking for destination route; they sustain a table utilized for this target. For this reason, they are called tabledriving routing protocols. The optimized link state routing (OLSR) protocol, with a first version that was defined in 2003 [19], while in 2014 second version was proposed.

In OLSR, routing tables in all nodes is used for preservation the routing information toward each other network's node for offering a destination route directly when chosen [20]. A multipoint relays (MPRs) is used in OLSR routing protocol as a most important key concepts with a main purpose of forwarding the broadcast messages in the network. The MPRs are selected by mobile nodes among their surrounding neighbors, after that they rebroadcast only those messages which were received from nodes who are MPR chosen. Two types of control messages are mainly used by OLSR, periodic HELLO messages are the first while the topology control (TC) are second type. The HELLO messages can be used for realizing the information deals with link status or another meaning, it performs the neighbor detector tasking. The topology control (TC) messages can be utilized for decelerating information about the MPR purposing. Principally, OLSR protocol is suitable for networks with intensive and great size due to the MPR technique which performs completely in such environment [21]. The collection of MPR in OLSR routing protocol is shown in Figure 3.

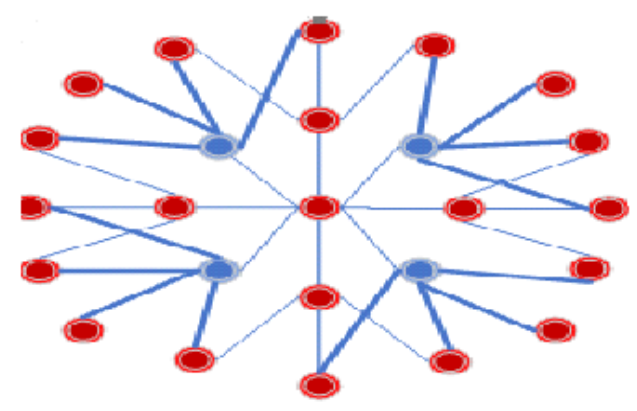

Figure 3. OLSR multipoint relay point

\subsection{Reactive routing protocol (on-demand)}

Reactive routing protocol waits for the demand prior to discover the packet destination route. Ad-hoc on-demand distance vector (AODV) considers the most applied with respect to all reactive protocols. This protocol is totally dissimilar to table-driven routing, On-demand protocol creates routes only if a request 
to send data is wanted. The mobile nodes Routing information does not keep by protocols as a substitute the route request is introduced only if needed, this means that if a source node need for create a route to the target, now the route will be started for assisting the source nodes request [22]. AODV is characterized as a pure on demand protocol structure, if a route is needed in the network, then an AODV implements a process of route discovery for finding the favorite destination route. Once the route is generated, it will be kept given that it is still desired by means of a route maintenance process.

AODV considers as a reactive on-demand routing protocol, in spite of reality of employing some features of the proactive routing approaches. AODV may be considered a good routing choice if a network behaves in dynamically changing. It combines the interesting features of both routing of DSR and DSDV with employing route maintenance besides route discovery concepts as a DSR protocol and using a sequence numbers in addition to transmit periodic hello messages return to DSDV protocol at the same time. The destination sequence numbers that using assures routes of loop-free and support available fresh routes detecting that permit the source nodes for finding out new routes every time [23]. AODV protocol can be used at least four control packets such as HELLO messages, route error messages (RERRs), route requests (RREQs), and route replies (RREPs) [24]. Every nodes in AODV are capable of routing table preserving that keeps all the information concerning the active routes. Such information represents the number of hops, next hop, destination's sequence number, routes active neighbors, and finally destination and expiration time related to a route table entry as shown in Figure 4.

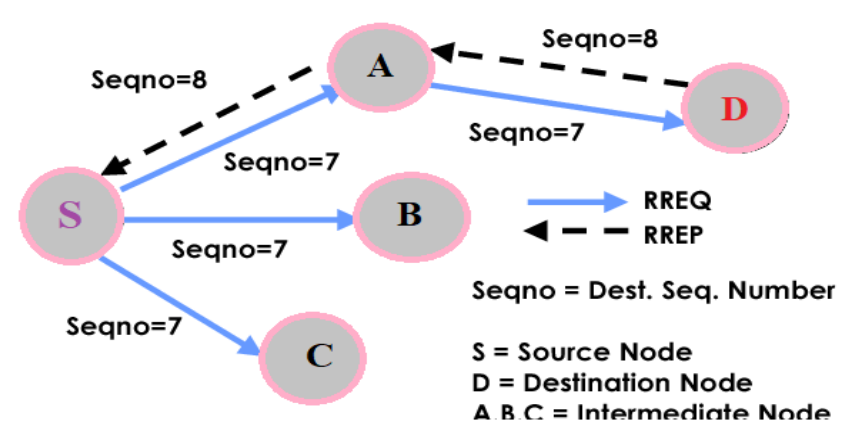

Figure 3. AODV process

\subsection{DSR (dynamic source routing)}

DSR is one of the most efficient routing protocols which can be used specifically in multi-hop MANET networks. It contains two phases: Route Discovery and Route Maintenance and they support nodes for finding and keeping the best destinations of the source routes. These source routings are loop-free routing that intermediate nodes require no current routing information and permit the nodes to store routing information for later use [25]. DSR considers as self-determining routing protocol that can be defined as a totally self-organizing self-configuring routing protocol. The discovery process can be improved in the network as routes caches are reserved at the mobile nodes and can be updated normally. DSR contains no data packets inside the network with any form at any level. As there was no declaration of the periodic routing, there were a using of neighbor detection or sensing packets of the link status in DSR.

\section{RELATED WORKS}

Wireless Ad hoc Networks consider dynamic with self-conFigured, in addition to be multi-hop networks, that have no infrastructure and centralized control forming. Here, some of the work related that summarizes a MANET routing protocols based on energy consumption for many researchers. Network Simulator- 2 (NS-2) and NS-3 are used to evaluate these protocols. Researchers find that a reactive routing DSR and AODV achieve well than table-based protocols OLSR. The mobility influence on energy conservation concerning these routing protocols presented by Chen Chang [26], with a result of reactive protocols consider more speed-sensitive while proactive protocols are not. But, if the nodes approach groups, the performance of on-demand protocols are better comparing to the proactive protocols based on energy conservation.

The first paper that compares between OLSR and DSR in terms of energy consumption can be found in [27]. Nitnaware, et.al found relation between routing policy and traffic rate in DSR, the low traffic 
rate gives good advantage in routing policy; but, with a high traffic rate, OLSR is performed better. Also, several researchers make an attempt for improving to the OLSR energy-efficiency [28]. Kafhali et al. made a comparison of the energy consumption to many protocols like AODV, DSR and DSDV based on three mobility as well as three traffic models [29]. The simulation gives a consideration that a total consumed energy (in joule) will be spent for transmitting and receiving of the control packets and a main conclusion is that AODV protocol has more energy consumption compared with DSR and DSDV in terms of CBR traffic.

Recently, a confirmation on comparing proactive, reactive and hybrid routing protocols has been existing. But practically a focusing on well-known performance metrics is done like throughput, end-to-end delay, and PDR. Samo, S.D. et al. consider energy in his work [30], they considered the throughput as well as the delay are addition to static and mobile scenarios, they also concentrated on the node remaining energy, but all the nodes are not with same energy consumption arrangement in the networks. Nitnawale et. al. [31] focus on an evaluation of various protocols based on Pareto traffic. they analyze on how to improve the consumed energy of routing protocols on their studies with different parameters taken into consideration. In the current paper, we have evaluated and analyzed the energy consumption of three prominent MANET routing protocols (AODV, OLSR and DSR) under constant bit rate CBR traffic in regards to different number of mobile nodes.

\section{NS-3 SIMULATION SET-UP OF ENERGY CONSUMPTION \\ 4.1. Simulation setup}

A Network Simulator (NS-3) is applied to simulate the three routing protocols. The protocols execution is completed in NS-3 and the performance of the proposed protocols are compared. NS-3 simulator is considered as discrete-event network designed for Internet systems specially for using in research and developments. The models of simulation are appropriately accurate for allowing to the NS-3 simulator to be considered as a real-time network's emulator. A real-time scheduler can be supported for allowing users to transceiver generated packets in NS-3 on real network devices in addition to be assist like interconnection framework between virtual machines. In this manuscript, an overall methodology for analyzing the energy consumption behavior in MANET within different scenarios. A node number of 5 to 20 have been chosen in the simulations, and the energy model will be same, but the server node considered the last nodes in the simulated network of the proposed protocols. The simulation parameters that are proposed for this simulation is shown in Table 1.

Table 1. The proposed simulation parameters

\begin{tabular}{cc}
\hline Parameters & Values \\
\hline Number of packets & 10000 \\
System type & MANET \\
Number of nodes & 5,20 \\
Simulation metrics & Energy, no. of packets \\
Protocol & OLSR \\
Interval & $4.1 \quad \mathrm{~s}$ to $0.9 \mathrm{~s}$ \\
\hline
\end{tabular}

\subsection{Metrics performances}

The total energy consumption by each node can be evaluated as sum of both transmitted and received energy intended for control packets. A transmitted energy required and the received in joules of the packets can be used these equations given below.

Transmitted Energy:

Trans. Energy= $($ TxPowerXPacket Size $) / 2 \times 106$

Receiving Energy:

Rec. Energy= $($ RxPowerXPacket Size $) / 2 \times 106$

Whereas the designed metrics of performances are focused on throughput besides end-end delay. Throughput that can be considered as a total byte's number per second evaluated in destination sinks. This means that can be calculated as the average packet's number received per amount of time. 
Throughput=(Total bytes received $* 8) /($ Last packet received-First packet received $)$

While the end-end delay denotes to the difference time interval for the packet origination to the final destination time when the packet reached the destination. Its calculated as:

(E-E) Delay=((Sum of the each CBR packet delays received $) / C B R$ packets number received $)$.

\section{RESULTS AND ANALYSIS}

The simulation results of presenting the routing protocols performances of any network scenarios relative to energy consumption will be explained according to several matrices. The control packets number which either generated or transmitted would be increased exponentially with the lower interval is, another thing if the interval time is as lower as 0.03 seconds, then a client is not eligible to generate the 10000 packets within a simulation duration of 500 seconds.

\subsection{Energy nodes with number of nodes}

The total energy consumed by the total participated nodes can be shown in Figure 4 for the control packets transmitted and received with a change in the mobile nodes number in the network. An increased node from 10 to 40 of the node densities at same network size for each routing protocol simulation's run is achieved. From Figure 4, it conforms that all routing protocols have an increased consumed energy with the mobile nodes number increasing which leads to bigger network size due to the network mobile nodes must processing all the routing packets. It can be seen from Figure 4 that, the consumed energy of all proposed protocols (DSR, AODV and OLSR) have quite similar from 10 to 20 nodes, but then a significant changing in energy consumption will be observed as the network size be bigger up to reach 40 nodes.

Specifically, the AODV protocol performance (on-demand routing) represented by energy consumption considered quite poor when comparing with DSR protocol, this is mainly because of increasing in the process of maintenance as increasing of nodes number in spite of both on-demand protocols (DSR and AODV) have similar behavior. It seems that AODV has an outstanding energy consumption with associated to DSR if the AODV process of maintenance considers as the main reason for this increasing.

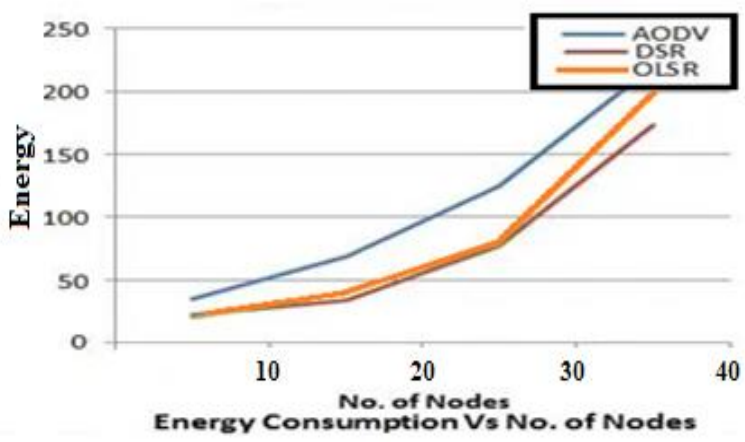

Figure 4. Energy consumption versus no of nodes

\subsection{Control Packets analysis with mobile nodes}

The Mac layer transmitting packets consider to be high compared with a transmitted packets, but essentially in the physical layer arrives 10000 packets generated. At the beginning of the time intervals, there are only 8000 transmitted packets because of using wireless network that uses a CSMA/CA mechanism with a collision avoidance happened as shown in Figure 5. This means that when the packets are generated totally, the nodes must be waited until there is a possible of transmission before transmittied and colliding.

The energy consumed is completely proportional to the transmitting packets by the sender (client) and the consumption happened only when packets are transmitting as shown in Figure 6. So, it is predicted that the total consumed network energy is same as a single node consumed energy which considered a client consumed energy. From Figure 7, the transmitted packets with 20 nodes started at high energy and then drop at the end of the interval due to many nodes are sending traffic therefore, the network saturation will be appeared earlier. The behavior of the energy is exactly same to the 5 nodes, a same curve mechanisim for the transmitted and received packets by the sender. 


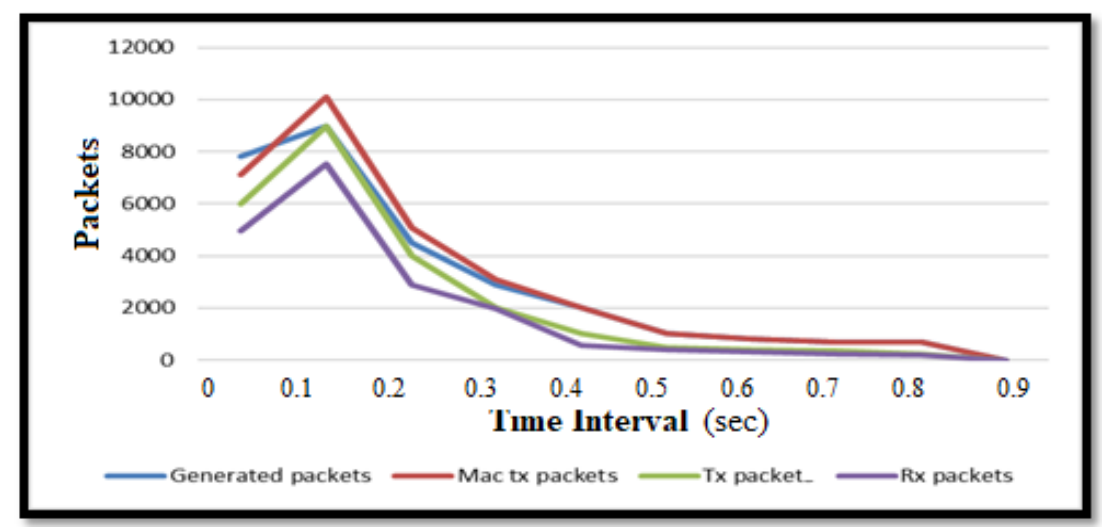

Figure 5. Packets analyzed with interval of 0.9

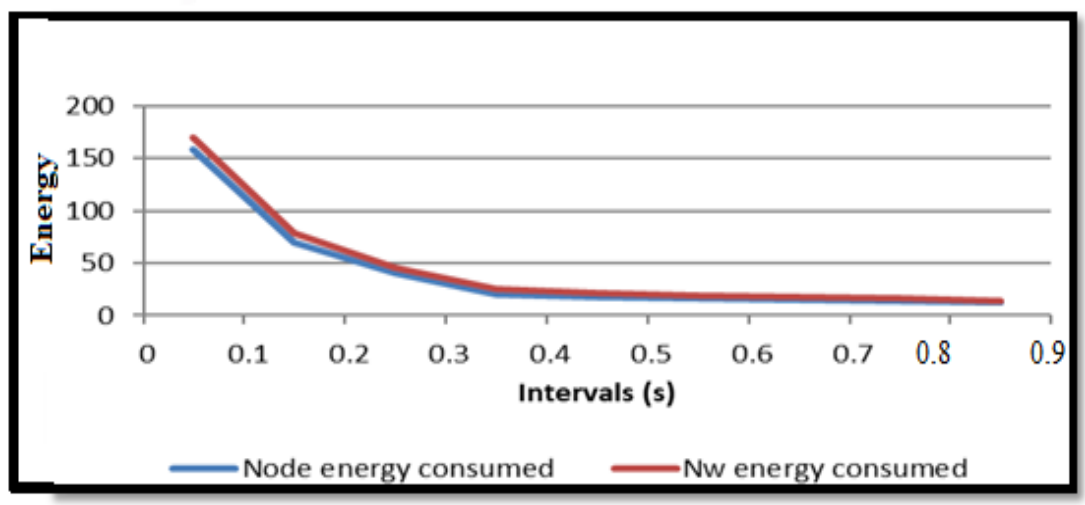

Figure 6. Packets analyzed with 5 nodes

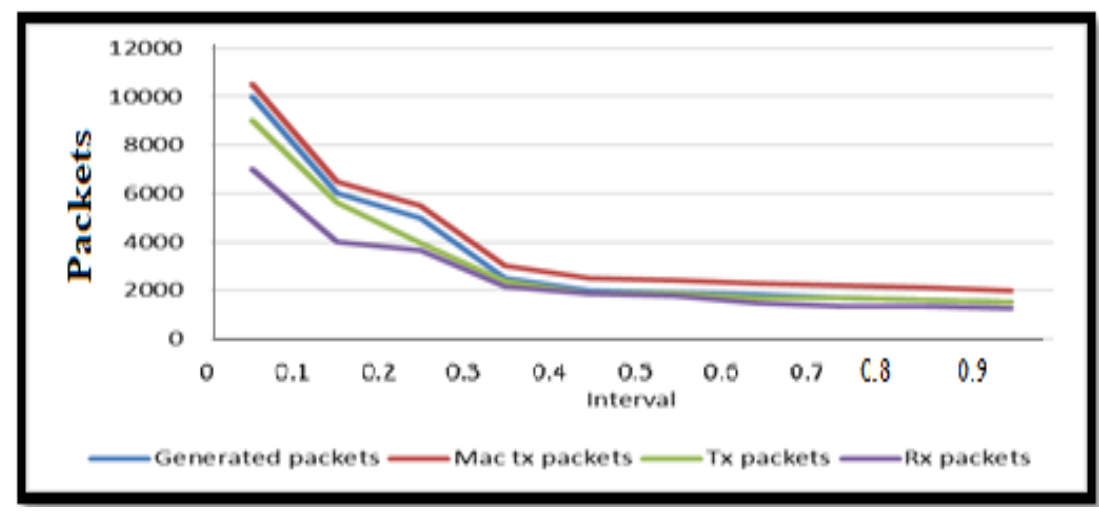

Figure 7. Packets analyzed with 20 nodes

\subsection{Consumed energy analysis}

The energy consumed with the mobile nodes is proportional to the transmitted control packets, thus when the mobile nodes are increasing to about 20, there is a gap in energy consumption occurred at the start of the simulation. The energy consumption of both routing protocols will be increased relatively at the identical pattern with increasing nodes number. Energy consumptions of the proposed routing protocols is showed in Figure 8. The reactive routing protocols represented by AODV and DSR have a little influenced by with the mobility compared with OLSR proactive routing protocols, so the two reactive routing protocols are more energy consumption than OLSR routing protocol regardless of the node's mobility. 


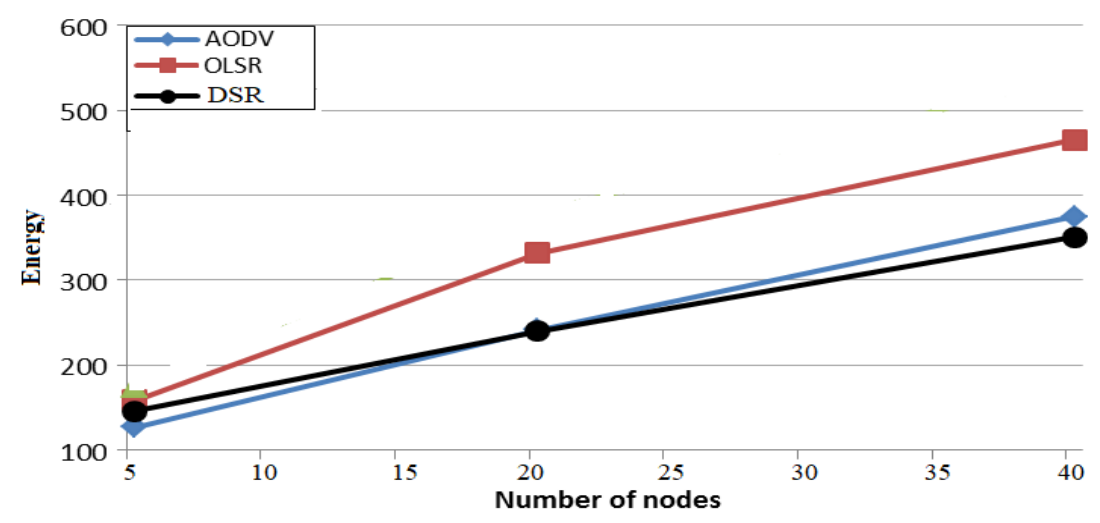

Figure 8. Energy consumptions of the proposed routing protocols

So, a node is consumed its battery and this leads to be disconnected from the network can be prevent with the proportionality of control packets transmitting, but with very high traffic cases, a mobile node would appear to loss connection with a network due to high collisions number suffering, thus a node will not able to transmit any packets when the network be continuously colliding.

\section{CONCLUSION}

This paper is presented the energy consumption of MANET routing protocols with various scenarios, and seen that the consumed energy considered as a direct consequence of the packets sent and received number. Hence, the energy management improvement may be realized for the modification and henceforth that considered for the MANETs effective energy protocols in which several applications are available, with a fast growing in MANETs nowadays. The controlling of the energy consumed can be depended on controlling of packets and their influence with the nodes number in the network which affects the traffic. The transmitted routing packets were increased as the total nodes number in the network, result a greater flooding for greater network.

\section{REFERENCES}

[1] R. Kumawat, Vinay Somani, "Comparative Study of On-demand Routing Protocols for Mobile Ad-hoc Network," vol. 27, no. 10, pp. 6-11, 2011.

[2] Y. H. Robinson, et al., "FD-AOMDV: Fault-tolerant disjoint ad-hoc on-demand multipath distance vector routing algorithm in mobile ad-hoc networks," J. Ambient Intell. Humaniz. Comput., pp. 4455-4472, 2019.

[3] Adnan H. Ali, Hayder J. Alhamdane, Begared S. Hassen, "Design analysis and performance evaluation of the WDM integration with CO-OFDM system for radio over fiber system," Indonesian Journal of Electrical Engineering and Computer Science, vol. 15, no. 2, pp. 870-878, Aug. 2019.

[4] R. Anand and J. Mungara, "Wireless Environment Aware Adaptive Scheduling Technique for Cellular Networks," Indones. J. Electr. Eng. Comput. Sci., vol. 11, no. 1, pp. 318-332, 2018.

[5] G. Chen, J. P. Coon, and S. E. Tajbakhsh, "Secure routing for multihop ad hoc networks with inhomo-geneous eavesdropper clusters," IEEE Trans. Veh. Technol., vol. 67, no. 11, pp. 10660-10670, 2018.

[6] M. M. Kareem, et al., "Grid Based Clustering Technique in Wireless Sensor Network using Hierarchical Routing Protocol," 2018 IEEE 4th International Symposium on Telecommunication Technologies (ISTT), pp. 1-5, 2018. doi: 10.1109/ISTT.2018.8701720.

[7] H. N. Saad, F. Jafar, and H. A. Salman, "A new compression technique in MANET : compressed-LZW algorithm," Indones. J. Electr. Eng. Comput. Sci., vol. 16, no. 2, pp. 890-896, 2019.

[8] A. Ambekar and H. D. Schotten, "Enhancing channel reciprocity for effective key management in wireless ad-hoc networks," IEEE 79th Vehicular Technology Conf. (VTC Spring), pp. 1-5, 2014.

[9] M. A. Saad, et al., "Spectrum sensing and energy detection in cognitive networks," Ind. J. Electr. Eng. Comput. Sci., vol. 17, no. 1, pp. 465-472, 2020.

[10] C.-C. Chang and H.-D. Le, "A provably secure, efficient, and flexible authentication scheme for ad hoc wireless sensor networks," IEEE Trans. Wirel. Commun., vol. 15, no. 1, pp. 357-366, 2016.

[11] D. Jiang, Z. Xu, and Z. Lv, "A multicast delivery approach with minimum energy consumption for wireless multihop networks," Telecommun. Syst., vol. 62, no. 4, 2015.

[12] Anas A. Hussien, Adnan H. Ali, "Comprehensive investigation of coherent optical OFDM-RoF employing 16QAM external modulation for long-haul optical communication system," International Journal Electrical and Computer Engineering (IJECE), vol. 10, no. 3, pp. 2607-2616, 2020, 
[13] Y. Neeraja and V. Sumalatha, "Collision-aware cooperative MAC protocol design for mobile ad-hoc networks," Indones. J. Electr. Eng. Comput. Sci., vol. 14, no. 1, pp. 455-461, 2019.

[14] M. Hadded, P. Muhlethaler, A. Laouiti, and L. A. Saidane, "A novel angle-based clustering algorithm for vehicular ad hoc networks," in Vehicular Ad-Hoc Networks for Smart Cities, Springer, pp. 27-38, 2017.

[15] Adnan H. Ali, Farhood, A. D., "Design and Performance Analysis of the WDM Schemes for Radio over Fiber System with Different Fiber Propagation Losses," Fibers, vol. 7, no. 3, pp. 19, 2019.

[16] D. Qin and Z. Ding, "On Transport Capacity of Full Duplex Ad Hoc Networks," 2015 IEEE Global Communications Conference (GLOBECOM), pp. 1-6, 2015. doi: 10.1109/GLOCOM.2015.7417311.

[17] C. Dong, et al., "Multicast in multi-channel cognitive radio ad hoc networks: Challenges and research aspects," Comput. Commun., vol. 132, pp. 10-16, 2018.

[18] C. Science, et al., "Link-state QoS routing protocol under various mobility models," Ind. J. Electr. Eng. Comput. Sci., vol. 16, no. 2, pp. 906-916, 2019.

[19] A.H. Farhood, A.D., Naji, M.K., Rhaif, S.H., Ali, "Design and analysis of dual band integrated hexagonal shaped microstrip UWB antenna," Indonesian Journal Electrical Engineering and Computer Science (IJEECS), vol. 15, no. 1, pp. 294-299, 2019.

[20] N. B. Gayathri, G. Thumbur, P. V. Reddy, and Z. U. R. Muhammad, "Efficient pairing-free certificateless authentication scheme with batch verification for vehicular ad-hoc networks," IEEE Access, vol. 6, pp. 31808$31819,2018$.

[21] M. K. Naji, A. D. Farhood, Adnan H. Ali, "Novel design and analysis of RF MEMS shunt capacitive switch for radar and satellite communications," Indonesian Journal of Electrical Engineering and Computer Science (IJEECS), vol. 15, no. 2, pp. 971-978, 2019.

[22] L. A. Khalil, A. Dulaimi, R. B. Ahmad, N. Yaakob, and M. H. Yusoff, "Wireless Environment Aware Adaptive Scheduling Technique for Cellular Networks,” Indones. J. Electr. Eng. Comput. Sci., vol. 13, no. 2, pp. 677-682, 2019.

[23] K. Lin, et al., "Virtualized QoS-Driven Spectrum Allocation in Space-Terrestrial Integrated Networks," IEEE Netw., vol. 33, no. 1, pp. 58-63, 2019.

[24] A. Kakkar, M. Singh, and A. Kakkar, "Robust authenticated encryption scheme with multiple keys for ad hoc networks,” Indones. J. Electr. Eng. Comput. Sci., vol. 15, no. 1, pp. 421-426, 2019.

[25] H. M. Noman, Ali A. Abdulrazzaq, Marwah M. Kareem, and Adnan H. Ali, "Improvement Investigation of the TCP Algorithms with Avoiding Network Congestion Based on OPNET," 2nd ICSET 2019, IOP Conf. Series: Materials Science and Engineering, vol. 518, no. 5, 2019.

[26] Samir A. Lafta, et al., "Performance simulation of broadband multimedia wireless networks simulation based on OPNET," IJEECS, vol. 17, no. 1, pp. 1-9, 2020. DOI: 10.11591/ijeecs.v17.i2.pp 1-9.

[27] T. Kunz, "Energy-Efficient Variations of OLSR," 2008 International Wireless Communications and Mobile Computing Conference, Crete Island, pp. 517-522, 2008. doi: 10.1109/IWCMC.2008.90.

[28] Maan, F. and Mazhar, N., "MANET Routing Protocols vs Mobility Models: A Performance Evaluation," $20113 \mathrm{rd}$ International Conference on Ubiquitous and Future Networks (ICUFN), pp. 179-184, 2011.

[29] Kafhali, S.E.L. and Haqiq, A., "Effect of Mobility and Traffic Models on the Energy Consumption in MANET Routing Protocols," International Journal of Soft Computing and Engineering (IJSCE), vol. 3, no. 1, pp. 242-249, 2013.

[30] Samo, S.D. and Fendji, J.L.E.K., "Evaluation of Energy Consumption of Proactive, Reactive, and Hybrid Routing Protocols in Wireless Mesh Networks Using 802.11 Standards," Journal of Computer and Communications, vol. 6, no. 4, pp. 1-30, 2018.

[31] Dhiraj Nitnaware Praveen Karma, Ajay Verma, "Performance Evaluation of Energy Consumption of Reactive Protocols under Self-Similar Traffic," International Journal of Computer Science \& Communication, pp. 305-306, 2010. 